\title{
An Axiomatic Specification for Sequential Memory Models
}

\author{
William Mansky, Dmitri Garbuzov, and Steve Zdancewic. \\ University of Pennsylvania
}

\begin{abstract}
Formalizations of concurrent memory models often represent memory behavior in terms of sequences of operations, where operations are either reads, writes, or synchronizations. More concrete models of (sequential) memory behavior may include allocation and free operations, but also include details of memory layout or data representation. We present an abstract specification for sequential memory models with allocation and free operations, in the form of a set of axioms that provide enough information to reason about memory without overly constraining the behavior of implementations. We characterize a set of "well-behaved" programs that behave uniformly on all instances of the specification. We show that the specification is both feasible - the CompCert memory model implements it - and usable - we can use the axioms to prove the correctness of an optimization that changes the memory behavior of programs in an LLVM-like language.
\end{abstract}

Keywords: memory models, optimizing compilers, deep specifications

\section{Introduction}

When reasoning about compilers and low-level code, it is not enough to treat memory as an assignment of values to locations; memory management, concurrency behavior, and many other factors complicate the picture, and without accounting for these factors our reasoning says nothing about the programs that actually run on processors. Memory models provide the necessary abstraction, separating the behavior of a program from the behavior of the memory it reads and writes. There have been many formalizations of concurrent memory models, beginning with sequential consistency [1] (in which memory must behave as if it has received an ordered sequence of read and write operations) and extending to more relaxed memory models. Most of these models include a theorem along the lines of "well-synchronized programs behave as if the memory model is sequentially consistent," characterizing a large class of programs that behave the same regardless of the concurrent memory model [7].

What, then, is the behavior of a sequentially consistent memory model? When the only memory operations are reads and writes (and possibly synchronization operations), the answer is simple: each read of a location reads the value that was last written to that location. In other words, the memory does in fact act as an assignment of values to locations. If we try to model other memory 
operations, however, the picture becomes more complicated. C and many related intermediate and low-level languages include at least allocation and free operations, and we might also want to include casts, structured pointers, overlapping locations, etc. Even restricting ourselves to sequential memory models, we can see that the space of possible models is much larger than "sequential consistency" suggests.

Formalizing memory models is a crucial step in compiler verification. Projects such as CompCert [4, CompCertTSO [9, Vellvm [11, and Compositional CompCert [8] specify memory models as part of the process of giving semantics to their various source, target, and intermediate languages, and use their properties in proving the correctness of optimizations and program transformations. The (in most cases sequential) memory models in these works include some of the complexity that more abstract formalisms lack, but they are also tightly tied to the particular languages and formalisms used in the projects. Compiler verification stands to benefit from memory model specifications that generalize away from the details of particular memory models, specifications which encompass most commonly used models and allow reasoning about programs without digging into the details of particular models. Generic specifications of memory models have the potential to lead to both simpler proofs - since all the reasoning about a particular model is encapsulated in a proof that it satisfies the specification - and more general ones - since a proof using a specification is true for any instance of that specification.

In this paper, we develop a specification for sequential memory models that support allocation and free operations as well as reads and writes, and demonstrate its use in reasoning about programs. We prove a sequential counterpart to the "well-synchronized programs are sequentially consistent" theorem, characterizing the set of programs that have the same behavior under any sequential memory model that meets our specification. We also show that CompCert's memory model is an instance of our specification, and verify a dead code elimination optimization for an LLVM-like language using the specification, resulting in a proof that is measurably simpler than the corresponding proof in Vellvm. All definitions and proofs have been formalized in the Coq proof assistant, so that our specification can be used for any application that requires mechanized proofs about programs with memory; the Coq development can be found online at http://www . seas . upenn. edu/ wmansky/meminterface

\section{An Abstract Sequential Memory Model}

A memory model is a description of the allowed behavior of a set of memory operations over the course of a program. A memory model can be defined in various ways: as a set of functions that can be called along with some guarantees on their results, as a description of the set of valid traces of operations performed by the execution of a program, or as an abstract machine that receives and responds to messages. In each case, the memory model makes a set of operations available to programs and provides some guarantees on their behavior. These 
operations always include reading and writing, and in many models these are the only operations; however, there are many other memory-related operations used in real-world programs. The main question is one of where we draw the line between program and memory. Is the runtime system that handles memory allocation part of the memory, or a layer above it? Does a cast from a pointer to an integer involve the memory, or is it a computation within the program? Does the memory contain structured blocks in which different references may overlap, or are structured pointers program objects that must be evaluated to references to distinct locations before they are read or written?

Our goal is to formalize the interface that memory provides to a programming language. We aim to give an abstract specification for memory models that can be used to define the semantics of a language, and to prove useful properties of programs in that language independently of the implementation details of any particular memory model. Our specification should describe the assumptions about memory that programmers can make when writing their programs and verifiers can make while reasoning. Since from the program's perspective the runtime system and the memory model are not distinct, our specification should include the operations provided by the runtime system. It should be easy to use in defining operational semantics for programming languages, and it should provide as many axioms as are needed to make the behavior of memory predictable without overconstraining the set of possible implementations.

For our specification, we begin with four operations: read, write, alloc, and free. These operations appear in code at almost every level. They are, for instance, the operations supported by the CompCert memory model [5], which has been used to verify a compiler from $\mathrm{C}$ to machine code. Although CompCert's model provides a realistic and usable formalization of the semantics of these operations, it is not the only such formalization. Other choices, such as CompCertTSO's [9] or the quasi-concrete model [2], may allow more optimizations on memory operations or a cleaner formulation of some theorems. We may want to store values in memory other than those included in CompCert, or abstract away from the details of blocks and offsets.

Our aim is to give a simple specification of memory models such that:

- Most memory models that support read, write, alloc, and free can be seen as instances of the specification.

- The specification provides the guarantees on these operations needed to reason about programs.

Then we can use this specification to reason about programs independently of the particular memory model being used, and by proving that particular models (such as CompCert's) meet the specification, be assured that our reasoning is valid for those models.

\subsection{Memory Model Axioms}

Previously, we mentioned three main approaches to specifying memory models. In the functional approach (e.g. CompCert [5]), each operation is a function with 
its own arguments and return type, and restrictions are placed on the results of the functions. In the abstract-machine approach (e.g. CompCertTSO [9]), memory is a separate component from the program with its own transition system, and steps of the system are produced by combining program steps and memory steps. In the axiomatic approach (taken in most concurrent memory models), a set of rules are given that allow some sequences of memory operations and forbid others. A definition in one of these styles is often provably equivalent to a definition in another style, although the axiomatic approach can be used to formalize some models that cannot be expressed in other ways (i.e. non-operational models). Our axioms should be true for all (reasonable) memory models, and also provide enough information to prove useful properties of a language that uses the specification.

Our model begins with a set $\mathcal{L}$ of locations and a set $\mathcal{V}$ of values. Every memory operation targets exactly one location, and locations are distinct: we can check whether two locations are equal, and a change to one location should not affect any other location. Locations may be thought of as unique addresses or memory cells. Values are the data that are stored in the memory; for simplicity, each location is assumed to be able to hold a single value of any size (in future work, we intend to extend this model to account for the size of data).

Definition 1. Given a location $\ell \in \mathcal{L}$ and a value $v \in \mathcal{V}$, a memory operation is one of $\operatorname{read}(\ell, v)$, write $(\ell, v)$, alloc $(\ell)$, and free $(\ell)$. The operations write $(\ell, v)$, alloc $(\ell)$, and free $(\ell)$ modify the location $\ell$. Over the course of execution, a program produces a series of memory operations. A memory model can be given as a predicate can_do on a sequence of memory operations $m=o p_{1} \ldots o p_{k}$ (called the history) and an operation $o p$, such that can_do $(m, o p)$ holds if and only if, given that the operations in $m$ have occured, the operation $o p$ can now be performed. A sequence of operations $o p_{1} \ldots o p_{k}$ is consistent with a memory model if can_do $\left(o p_{1} \ldots o p_{i-1}, o p_{i}\right)$ for each $i<k$, i.e., each operation in the sequence was allowable given the operations that had been performed so far.

The axioms shown in Figure 1 restrict the possible behavior of a can_do predicate. (We write $l o c(o p)$ for the location accessed by $o p$.) The first two axioms state the distinctness of locations, requiring that operations on one location do not affect the operations possible on other locations. The remaining rules enforce (but do not completely determine) the intended semantics of each kind of memory operation: e.g., a write $(\ell, v)$ operation must allow $v$ to be read at $\ell$. We do not completely constrain the semantics of the operations, but we attempt to capture the expectations of a programmer about each operation: it should be possible to allocate free memory and free allocated memory, write to allocated memory and read the last value written, etc., and it should not be possible to free memory that is already free, allocate memory that is already allocated, read values that have not been written, etc. Note that, while the axioms are meant to define the possible semantics of memory models, they also coincide with the sorts of equivalences that are commonly used in compiler optimizations - reordering unrelated operations, propagating stored values forward to later reads, etc. 


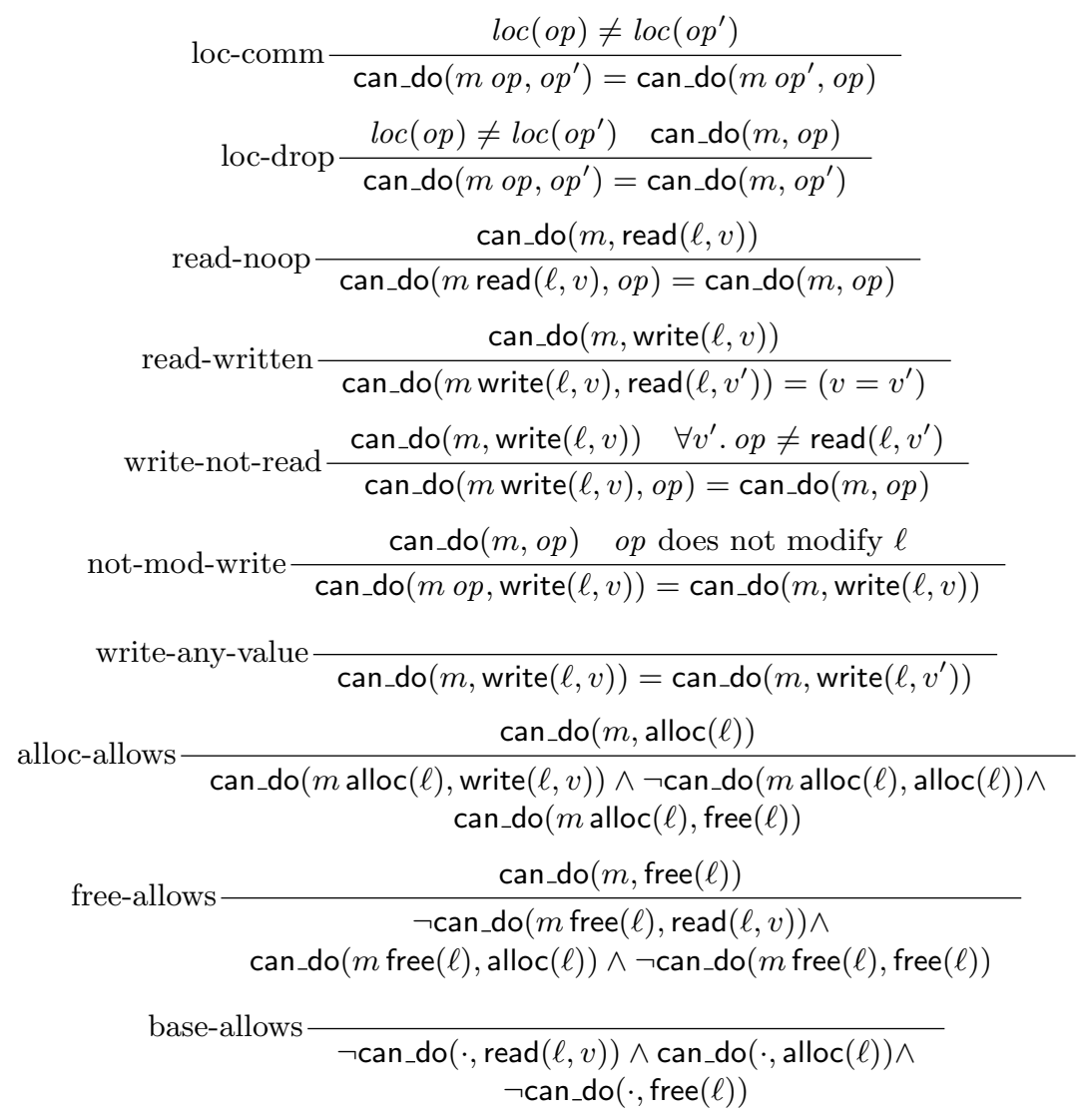

Fig. 1. The axioms of the memory model specification

If a behavior is "implementation-dependent", or might vary across different memory models, then the axioms leave it unspecified. Two major kinds of operation are left unspecified: reads from locations that have been allocated but not written to (we call these locations "uninitialized"), and writes to locations that have not been allocated. Because these operations are unspecified, the specification admits instances in which they have a wide variety of interpretations: a write to an allocated location may fail, write a value that can be read later, unpredictably either allocate the location and write a value or do nothing at all, or any other (possibly empty) subset of the conceivable behaviors of a write, depending on the memory model. Parameterizing by the sets $\mathcal{L}$ and $\mathcal{V}$ also implicitly leaves some aspects of the memory model unspecified. We do not constrain the kinds or sizes of data that can be stored (although we do require that any value can be stored in any location and read back unchanged), and we do not specify whether there is a finite or an infinite number of locations. If we instantiate the specification with an infinite $\mathcal{L}$, then for any $m$ there is an $\ell$ such that can_do $(m$, alloc $(\ell))$; 
if we choose a finite $\mathcal{L}$, then we may reach states in which there is no such $\ell$. The effect of running out of memory on program executions is left to the language semantics, as we will show in Section 4.1.

Although each axiom only specifies the interaction between the new operation and the most recent operation performed, we can derive rules that connect each new operation to "the last relevant operation", e.g., the last alloc or free of a location being written. For instance, we can prove that if $m$ write $(\ell, v)$ write $\left(\ell^{\prime}, v^{\prime}\right)$ is a consistent history for some $m$, then $\operatorname{can} \_d o\left(m \operatorname{write}(\ell, v) \operatorname{write}\left(\ell^{\prime}, v^{\prime}\right), \operatorname{read}(\ell, v)\right)$ holds:

$$
\begin{array}{ll}
\text { can_do }\left(m \text { write }(\ell, v) \text { write }\left(\ell^{\prime}, v^{\prime}\right), \operatorname{read}(\ell, v)\right) & \\
=\text { can_do }(m \text { write }(\ell, v), \operatorname{read}(\ell, v)) & \text { by loc-drop } \\
=(v=v) & \text { by read-written } \\
=\text { true } &
\end{array}
$$

In each step, the condition that can_do holds on the operations in the history follows from the consistency assumption. In general, our rules only allow complex reasoning about histories if those histories are consistent; an unspecified operation may have unpredictable effects on memory behavior (e.g., a write to an unallocated location may or may not quietly cause that location to be allocated).

In the context of concurrent memory models, it is usually assumed or proved that well-synchronized programs are sequentially consistent, regardless of the relaxations allowed by the memory model. This allows the complexities of the model to be hidden from the programmer, and means that verification of a certain (large) class of programs can be done independently of the relaxed model. Our axiomatization admits a similar property for sequential memory models. Consider a simple abstract machine that associates each memory location with one of three states: free, uninit, or stored $(v)$, where $v$ is a value. Upon receiving a memory operation on a location, the machine's state for the location transitions as shown in the register automata of Figure 2 any operation not shown leads to an error state.

Definition 2. The simple machine corresponding to a history $m$, written $\mathrm{SM}(m)$, is the machine reached by starting with each location in the Free state and applying the operations in $m$ to their corresponding locations, in order. The can_do predicate induced by the simple machine is the one such that can_dosm $(m, o p)$ when $l o c(o p)$ has a transition labeled with op in $\operatorname{SM}(m)$.

Then we can prove the following theorems:

Theorem 1. The simple machine satisfies the memory model axioms.

Theorem 2. If a program never reads an uninitialized location and never writes to a free location, then for any can_do predicate that satisfies the axioms and any consistent history $m$ and operation op, can_do $(m, o p)$ if and only if can_dosm $(m, o p)$.

This gives us a class of programs for which any model that satisfies the axioms is equivalent. For the (large) set of programs that take a principled approach 


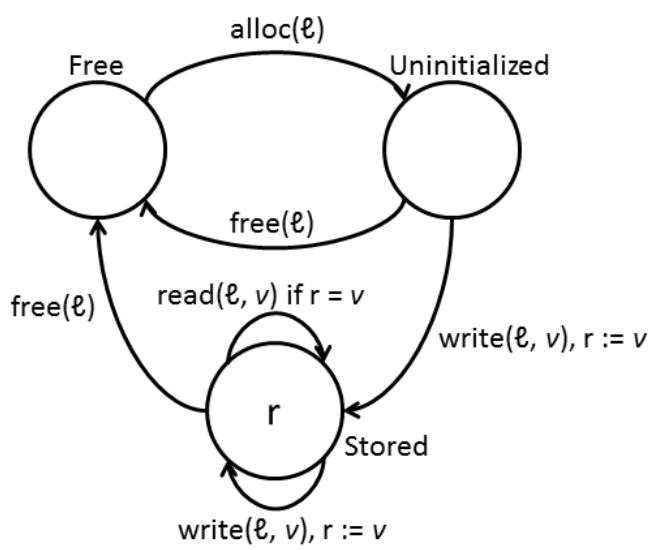

Fig. 2. The transition system for locations in the simple memory machine

to memory and avoid implementation-dependent behavior, we can reason using the simple machine and derive results that are applicable to any memory model that implements the specification; this has the potential to greatly simplify our proofs. On the other hand, many interesting programs may not meet the requirements of the theorem. In this case, we may still be able to reason using the specification: while we cannot turn to the fully defined simple machine, we can still use the axioms to draw conclusions about a program's memory behavior. Finally, if we expect that the correctness of our reasoning depends on a particular implementation, then we can go beneath the specification and work with the implementation directly. Having a reasoning principle for "well-behaved" programs simplifies our reasoning when it can be applied, but does not force us to give up on reasoning about programs that are not well-behaved.

\section{Instantiating the Specification}

The CompCert verified C compiler includes a C-like memory model [5], which is used to verify its transformations. In fact, it includes both a specification of a memory model and an implementation of that specification. Memory is modeled as a set of non-overlapping blocks, each of which behaves as an array of bytes; an address is a pair $(b, o)$ of a block and an offset into the array. The specification defines four functions that can be called by programs (alloc, free, load, and store) and states properties on them. Most of these properties center around the permissions associated with each address, such as Readable, Writeable, and Freeable, which indicate which operations can be performed on the address. Figure 3 shows some of the properties for store; the other operations have similar axioms. CompCert's memory implementation manages the bounds, allocation state, and content of each block in a way that is shown to satisfy the axioms. 


$$
\begin{gathered}
\frac{(b, o) \text { has permission Writeable in } M_{1}}{\exists M_{2} . \text { store }\left(M_{1}, b, o, v\right)=M_{2}} \\
\frac{\text { store }\left(M_{1}, b, o, v\right)=M_{2} \quad\left(b^{\prime}, o^{\prime}\right) \text { has permission } p \text { in } M_{1}}{\left(b^{\prime}, o^{\prime}\right) \text { has permission } p \text { in } M_{2}} \\
\text { store }\left(M_{1}, b, o, v\right)=M_{2} \quad\left(b^{\prime}, o^{\prime}\right) \text { has permission } p \text { in } M_{2} \\
\frac{\left(b^{\prime}, o^{\prime}\right) \text { has permission } p \text { in } M_{1}}{\text { store }\left(M_{1}, b, o, v\right)=M_{2}} \\
\hline(b, o) \text { has permission Writeable in } M_{1}
\end{gathered}
$$

Fig. 3. A few of CompCert's store axioms

Although the CompCert memory specification abstracts away from some of the details of the implementation, it has some limitations as a generic memory model specification. It is tied to CompCert's particular definition of values and its notion of blocks. Furthermore, there is no uniformity across the different memory operations; each function takes different arguments and has a different result type, so that 44 axioms are used to express properties of the sort laid out in our specification. The CompCert memory model specification does not include an axiom that says "operations on different locations are independent"; indeed, it is difficult to state such an axiom, since "operations" are not quantifiable objects. Instead, we can look at the axioms stating that, e.g., a store to $(b, o)$ does not change the permissions of another block and a free succeeds as long as the target address is Freeable, and conclude that a free can occur after a store to a different location if and only if it could occur before the store.

Using this sort of reasoning, we can show that the CompCert memory model specification satisfies our specification in turn. We "implement" each one of our memory operations with a call to the corresponding CompCert function, with one allocated block for each allocated memory location. Our specification does not include details about the size of values, so we restrict ourselves to 32-bit values (which includes most CompCert values).

Definition 3. Given CompCert memory states $M$ and $M^{\prime}$, let $M \stackrel{o p}{\rightarrow} M^{\prime}$ if the function call corresponding to $o p$ can be applied to $M$ to yield $M^{\prime}$. Let can_docc $(m, o p)$ be true when there exist CompCert memory states $M_{1}, M_{2}$ such that empty $\stackrel{m}{\longrightarrow}^{*} M_{1}$ and $M_{1} \stackrel{o p}{\longrightarrow} M_{2}$, where empty is the initial CompCert memory state.

Theorem 3. can_docc satisfies the axioms of our specification.

Proof. The difficult axioms are loc-comm and loc-drop, since the other axioms refer to the interaction of particular operations. For each of loc-comm and locdrop, we must break the proof into 16 cases, one for each ordered pair of memory operations. The cases involving load are straightforward, since it does not change the memory state. In each other case, we must show that the first operation does not change the permissions associated with the location of the second 
operation and vice versa. This allows us to conclude that each operation can still be performed after an operation to a different location is reordered/dropped.

This provides some evidence for the feasibility of our specification, since the CompCert memory model (when used in this restricted way) satisfies its axioms. By Theorem 2, we also know that on programs that do not read uninitialized locations or write to free locations, the CompCert memory model has the same behavior as the simple abstract machine. (The CompCert specification requires that reads of uninitialized locations return a special undef value and writes to free locations fail, which is just one point in the design space of memory models allowed by our specification; reads of uninitialized locations could also fail or return arbitrary values, for instance.)

Interestingly, while we choose the set of 32-bit CompCert values as our $\mathcal{V}$ for this instance, we do not need to choose a particular $\mathcal{L}$ in order to prove the above theorem. Each allocated location is mapped to a block, but the set of locations need not be the set of blocks itself. In the CompCert memory model, an alloc call always succeeds, implying that memory is infinite; however, the proof of implementation still applies even if we choose a finite $\mathcal{L}$. In this case, while CompCert's memory model is always willing to allocate more blocks, programs may still run out of distinct locations to request. Our specification's view of CompCert's infinite-memory model gives us an interface that can be either infinite-memory or finite-memory.

\section{Using the Specification}

From the perspective of a programming language, a memory model fills in the gaps in the semantics and provides some guarantees about the observable behavior of the memory. In this section, we show how our specification can be used for these tasks, by defining the semantics of a language using the specification and verifying an optimization against it.

\subsection{MiniLLVM}

Our example language is MiniLLVM, a language based on the LLVM intermediate representation [3]. The syntax of the language closely resembles LLVM, with the slight variation that labels are implicit in the structure of the control flow graph rather than explicitly present in the instructions.

$$
\begin{aligned}
& \operatorname{expr}::=\% \mathrm{x}|@ \mathrm{x}| \mathrm{c} \quad \text { type }:=\text { int } \mid \text { type pointer } \\
& \text { instr }::=\% \mathrm{x}=\text { op type expr, expr } \mid \% \mathrm{x}=\mathrm{i} \mathrm{cmp} \text { cmp type expr,expr } \mid \\
& \text { br expr | br | alloca \%x type | } \\
& \% \mathrm{x}=\text { load type }{ }^{*} \text { expr } \mid \text { store type expr, type* expr } \mid \\
& \% \mathrm{x}=\text { cmpxchg type }{ }^{*} \text { expr, type expr, type expr } \\
& \% \mathrm{x}=\operatorname{phi}\left[\text { node }_{1}, \operatorname{expr}_{1}\right], \ldots,\left[\text { node }_{k}, \operatorname{expr}_{k}\right] \mid \\
& \% \mathrm{x}=\text { call type expr }(\operatorname{expr}, \ldots, \text { expr }) \mid \text { return expr } \mid \text { output expr }
\end{aligned}
$$


A MiniLLVM program $P$ is a list of function definitions $(f, \ell$, params, $G)$, where $f$ is the name of the function, $\ell$ is its location in memory, params is the list of the function's formal parameters, and $G$ is the function's control-flow graph (CFG). (For simplicity, we assume that each node in a CFG contains exactly one instruction.) A configuration is either an error state Error or a tuple $\left(f, p_{0}, p, e n v, s t, a l\right)$, where $f$ is the name of the currently executing function, $p_{0}$ is the previously executed program point, $p$ is the current program point, env is the environment giving values for thread-local variables, st is the call stack, and $a l$ is a record of the memory locations allocated by the currently executing function (the alloca instruction allocates space that is freed when the function returns). The semantics of MiniLLVM are given by a transition relation $P \vdash c \stackrel{a}{\rightarrow} c^{\prime}$, where $a$ is either a list of memory operations performed in the step or a value output by the output instruction. A few of the semantic rules for MiniLLVM instructions are shown in Figure 4, where $P_{f}$ is the CFG for the function $f$ in $P, \operatorname{succ}(p)$ is the successor node of $p$ in its CFG, Label extracts the instruction label for a node from the CFG, and $(e, e n v) \Downarrow v$ means that the expression $e$ evaluates to $v$ in the presence of the environment env. We make a point of allowing the store instruction to fail into an Error state so that in our example optimization - a dead store elimination - we can safely remove ill-formed stores.

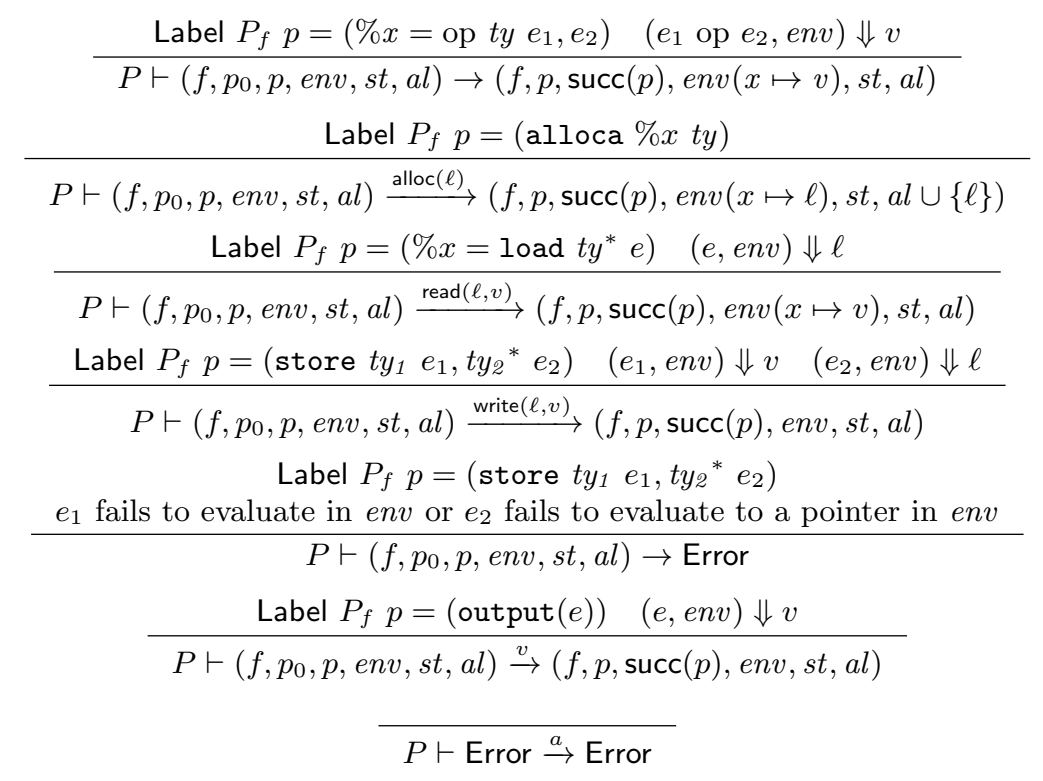

Fig. 4. Part of the transition semantics of MiniLLVM

Note that the interaction between the semantics of MiniLLVM and the memory model is restricted to the transition labels. We complete the semantics by combining the transitions of the language with an instance of the memory model 
specification, passing the memory operations to the can_do predicate and retaining the output values, if any:

$$
\text { mem-step } \frac{P \vdash c \stackrel{o p_{1}, \ldots, o p_{n}, v_{1}, \ldots, v_{k}}{\longrightarrow} c^{\prime} \quad \operatorname{can} \_d o\left(m, o p_{1} \ldots o p_{n}\right)}{P \vdash(c, m) \stackrel{v_{1}, \ldots, v_{k}}{\longrightarrow}\left(c^{\prime}, m o p_{1} \ldots o p_{n}\right)}
$$

So while, e.g., a load operation may produce read $(\ell, v)$ for any $v$, the only $v$ that will be allowed by the can_do predicate is the one stored at $\ell$. To obtain MiniLLVM semantics for a particular memory model, we simply instantiate the rule with the can_do predicate for that model; we can also reason at the level of the specification and derive results that hold for every instance.

Finite Memory Semantics In Section 2.1, we noted that our specification encompasses both infinite-memory and finite-memory models, and indeed our semantics for MiniLLVM works in either case. However, it is interesting to consider the way that finite memory is reflected in the semantics. If the set of locations is finite, then we may reach a state $(c, m)$ in which can_do $(m$, alloc $(\ell))$ does not hold for any $\ell$. In this case, the mem-step rule cannot be applied, and $(c, m)$ is stuck. In terms of optimizations, this means that alloca instructions may not be removed from programs, since this may enable behaviors that were previously impossible due to the out-of-memory condition.

An alternative approach is to treat out-of-memory as an error state. We can obtain this semantics by adding one more rule:

$$
\frac{P \vdash c \stackrel{\text { alloc }(\ell)}{\longrightarrow} c^{\prime} \quad \forall \ell . \neg \text { can_do }(m, \operatorname{alloc}(\ell))}{P \vdash(c, m) \rightarrow(\text { Error }, m)}
$$

Now the language semantics catches the out-of-memory condition and transitions to an error state rather than getting stuck. This new semantics allows alloca instructions to be removed but not inserted, since optimizations should not introduce new errors. (With a more sophisticated treatment of $\mathcal{L}$, we may be able to state a semantics that allows both adding and removing alloca.) We can choose whichever semantics is appropriate to the language or the application at hand; our specification implicitly makes the behavior of out-of-memory programs a question of language design rather than a feature of the memory model itself.

\subsection{Verifying an Optimization}

A good specification should allow us to abstract away from unnecessary details, so that we can separate reasoning about programs from reasoning about memory models. In this section, we will use the semantics of MiniLLVM with the memory model specification to prove the correctness of a dead store elimination optimization (under any memory model that satisfies the specification). We will assume that we have some analysis for finding dead stores, and prove that removing dead stores does not change the behaviors of a MiniLLVM program. 
To begin, we need to state our notion of correctness. A correct optimization should refine the behaviors of a program; it may remove some behaviors (e.g. by collapsing nondeterminism), but it should never introduce new behaviors.

Definition 4. A configuration is initial if it is a tuple $\left(f, p_{0}, p, e n v, s t, a l\right)$ such that $s t$ and $a l$ are empty and $p$ is the start node of $P_{f}$. A trace of a program $P$ is a sequence of values $v_{1}, \ldots v_{n}$ for which there is some initial configuration $c_{0}$ and some state $\left(c^{\prime}, m^{\prime}\right)$ such that $\left(c_{0}, \cdot\right) \stackrel{v_{1}, \ldots, v_{n}}{\longrightarrow}{ }^{*}\left(c^{\prime}, m^{\prime}\right)$. A program $P$ refines a program $Q$ if every trace of $P$ is a trace of $Q$.

We can prove refinement through the well-established technique of simulation. In particular, since dead store elimination removes an instruction from the program, we will use right-option simulation, in which the original program may take some externally unobservable steps that the transformed program omits.

Definition 5. A relation $R$ on states is a right-option simulation between programs $P$ and $Q$ if the initial states of $P$ and $Q$ are in $R$ and for any states $C_{P}, C_{Q}$ in $P$ and $Q$ respectively, if $R\left(C_{P}, C_{Q}\right)$ and $P \vdash C_{P} \stackrel{k}{\rightarrow} C_{P}^{\prime}$, then there is a state $C_{Q}^{\prime}$ such that $R\left(C_{P}^{\prime}, C_{Q}^{\prime}\right)$ and either

$$
\begin{aligned}
& -Q \vdash C_{Q} \stackrel{k}{\rightarrow} C_{Q}^{\prime} \text {, or } \\
& -\exists C_{Q}^{\prime \prime} \cdot Q \vdash C_{Q} \rightarrow C_{Q}^{\prime \prime} \text { and } Q \vdash C_{Q}^{\prime \prime} \stackrel{k}{\rightarrow} C_{Q}^{\prime} .
\end{aligned}
$$

Theorem 4. If there is a right-option simulation between $P$ and $Q$, then $P$ refines $Q$.

We conservatively approximate dead stores by defining them as stores to locations that will never be read again.

Definition 6. An instruction store $t y_{1} e_{1}, t y_{2}{ }^{*} e_{2}$ in a program $P$ is dead if in all executions of $P$, if $e_{2}$ is evaluated to a location $\ell$ when the store is executed, then $\ell$ will not be the target of a read for the remainder of the execution.

The optimization itself, given a dead store, is simple: we remove the node containing the dead store from its CFG. The simulation relation $R_{d s e}$ relates a state $\left(c^{\prime}, m^{\prime}\right)$ in the transformed program to a state $(c, m)$ in the original program if $m^{\prime}$ can be obtained from $m$ by dropping writes to locations that will not be read again, and $c^{\prime}$ can be obtained from $c$ by replacing the removed node $n$ with its immediate successor.

Definition 7. Let $P$ be a graph in which the function $f$ contains a node $n$ whose successor is $n^{\prime}$. The predicate skip_node holds on a pair of configurations $\left(c, c^{\prime}\right)$ if either both $c$ and $c^{\prime}$ are Error, or $c^{\prime}$ can be obtained from $c$ by replacing all occurrences of $n$ in the program point and the stack with $n^{\prime}$. Let $R_{d s e}$ be the relation such that $R_{d s e}\left(\left(c^{\prime}, m^{\prime}\right),(c, m)\right)$ when either

$-c=$ Error, or

- $m^{\prime}$ can be obtained from $m$ by removing writes to locations that will not be targeted by reads for the rest of the execution, and skip_node $\left(c, c^{\prime}\right)$ holds. 
The proof proceeds as follows. First, we show that any step in the transformed graph can occur in the original graph.

Lemma 1. Let $P^{\prime}$ be the program obtained from $P$ by removing a node $n$ from $a$ function $f$, and $n^{\prime}$ be the successor of $n$. If $P^{\prime} \vdash\left(c^{\prime}, m\right) \stackrel{k}{\rightarrow}\left(c_{2}^{\prime}, m_{2}\right)$, skip_node $\left(c, c^{\prime}\right)$, and $c$ is not at $n$, then there exists $c_{2}$ such that $P \vdash(c, m) \stackrel{k}{\rightarrow}\left(c_{2}, m_{2}\right)$ and $\operatorname{skip\_ node}\left(c_{2}, c_{2}^{\prime}\right)$.

Proof. Because $c$ is not at $n, c$ and $c^{\prime}$ execute the same instruction and produce the same results, modulo the fact that $n$ is present in $P$ and absent in $P^{\prime}$ (giving us skip_node $\left.\left(c_{2}, c_{2}^{\prime}\right)\right)$.

Next, we show that dropping writes to unread locations from a history does not change the operations it allows.

Lemma 2. Let $m$ and $m^{\prime}$ be consistent histories such that $m$ is produced by a partial execution of a program $P$ and $m^{\prime}$ can be obtained from $m$ by removing writes to locations that are not targeted by read $s$ for the rest of the execution. If $P$ never reads uninitialized locations or writes to free locations, then can_do $(m, o p)$ if and only if can_do $\left(m^{\prime}, o p\right)$.

Proof. By Theorem 2, can_do $(m, o p)$ iff $\operatorname{can} \_d o s m(m, o p)$ (and likewise for $m^{\prime}$ ). We can show by induction that for any location $\ell$, if $\operatorname{SM}(m)$ and $\operatorname{SM}\left(m^{\prime}\right)$ differ, then $\operatorname{SM}(m)$ is in the Stored state and $\operatorname{SM}\left(m^{\prime}\right)$ is not in the Freed state (and $\ell$ is not read again in the execution). This is sufficient to guarantee that any non-read operation has the same effect in $\operatorname{SM}(m)$ and $\operatorname{SM}\left(m^{\prime}\right)$, and the conclusion follows directly.

We can use this lemma to show that the relationship between memories is preserved by program steps.

Lemma 3. Let $m$ and $m^{\prime}$ be consistent histories such that $m$ is produced by a partial execution of a program $P$ and $m^{\prime}$ can be obtained from $m$ by removing writes to locations that are not targeted by reads for the rest of the execution. If $P$ never reads uninitialized locations or writes to free locations and $P \vdash\left(c, m^{\prime}\right) \stackrel{k}{\rightarrow}$ $\left(c_{2}, m_{2}^{\prime}\right)$, then there exists $m_{2}$ such that $P \vdash(c, m) \stackrel{k}{\rightarrow}\left(c_{2}, m_{2}\right)$ and $m_{2}^{\prime}$ can be obtained from $m_{2}$ by removing writes to locations that are not targeted by reads for the rest of the execution.

Proof. Since we never observe the differences between $m$ and $m^{\prime}$, we can take the same steps and produce the same operations under each history, preserving the relationship between them.

Lemmas 1 and 3 taken together, with a little reasoning about the effects of the dead store, allow us to conclude that $R_{d s e}$ is a simulation relation.

Theorem 5. Let $P^{\prime}$ be the program obtained from $P$ by removing a dead store, and suppose that $P^{\prime}$ never reads an uninitialized location and $P$ never writes to a free location. Then $R_{d s e}$ is a right-option simulation between $P^{\prime}$ and $P$, and so $P^{\prime}$ refines $P$. 
Proof. The combination of Lemmas 1 and 3 give us all cases except the one in which $P$ executes the removed store. In that last case, we can show that the effect of the store is to augment the history with a write to a location that is not the target of a read for the rest of the execution, and after executing the store, $P$ is once again in lockstep with $P^{\prime}$.

Note that since $P^{\prime}$ has fewer writes than $P$, it may have more uninitialized locations, and so the condition on reads must be checked on $P^{\prime}$ and the condition on writes must be checked on $P$. We can conclude that, for this class of wellbehaved programs, the dead store elimination optimization is correct under any memory model that meets the specification.

Comparison with Vellvm Using a more abstract specification should lead to simpler proofs, giving us a more concise formulation of the properties of the memory model and allowing us to avoid reasoning about details of the model. The Vellvm project [11] also included a dead store elimination for an LLVM-based language verified in Coq, using a variant of the CompCert memory model, and so provides us a standard with which to compare our proofs. While it is difficult to compare different proof efforts based on different formalizations, several metrics suggest that our specification did indeed lead to significantly simpler proofs. Vellvm's DSE verification consists of about 1860 lines (65k characters) of definitions and proof scripts, while our verification is 890 lines (44k characters). A separate section of Vellvm's code is devoted to lifting CompCert's memory axioms for use in the proofs - essentially the memory model specification for Vellvm - and this section is 1200 lines (38k characters), while our memory model specification is 420 lines (17k characters). To correct for the effects of different proof styles on line and character counts, we also compared the gzipped sizes of the developments; Vellvm's proof is 12.4 kilobytes, our proof is 8.3 kilobytes, Vellvm's specification is 6.7 kilobytes, and our specification is 3.3 kilobytes.

Although Vellvm's language is more featureful than MiniLLVM, this appears to account for little of the difference in the proofs, since most of these features are orthogonal to memory operations. Roughly speaking, our proof of correctness is $2 / 3$ the size of Vellvm's and our specification is half the size, supporting the assertion that our specification lends itself to simpler proofs. Furthermore, our results hold not just for one model but for any instance of the specification.

\section{Related Work}

There have been many efforts to generically specify concurrent and relaxed memory models. The work of Higham et al. [1] is an early example of formalizing memory models in terms of sequences of read and write events; this approach is used to formalize models ranging from linearizability to TSO and PSO. Yang et al. [10] gave axiomatic specifications of six memory models, and used constraint logic programming and SMT solving to check whether specific executions adhered to the models. Saraswat et al. [7] gave a simple specification for concurrent memory 
models in terms of the "well-synchronized programs are sequentially consistent" property, and demonstrated that their specification could be instantiated with both models that prohibited thin-air reads and those that allowed them. In all these works, reads, writes, and synchronizations were assumed to be the only memory operations, and thus "sequential consistency" was taken to uniquely define the single-threaded memory model.

Owens et al. 6] defined the x86-TSO memory model, and showed that their axiomatic definition was equivalent to an abstract-machine model. This model formed the basis for the memory model of CompCertTSO 9], the main inspiration for our work. CompCertTSO's model includes alloc and free operations, and we follow its approach in giving semantics to our language by combining language steps and memory steps. CompCertTSO does not seek to give a general specification of a category of memory models, but rather a single instance with TSO concurrency and CompCert-specific allocation and free behavior. We know of no other work that attempts to give a generic, language-independent specification of memory models with operations beyond read and write.

\section{Conclusions and Future Work}

While much work has gone into formalizing the range of possibilities for concurrent memory models, less attention has been devoted to a truly generic description of sequential memory models. Our specification is a first step towards such an account, and we have highlighted the properties of generality, feasibility, and usability that make it a reasonable specification for sequential memory models with allocation and free operations. We have characterized the set of programs for which all such models are equivalent, proved that CompCert's memory model is an instance of our specification, and used it to verify an optimization with proofs demonstrably simpler than those written without such a specification.

Our memory model specification is currently based on the simplifying assumption that the size of data does not matter. Reflecting the size of data in the specification (e.g. by specifying the size of each allocation and allowing reads/writes to offsets within blocks) would allow us to more faithfully model CompCert's and other C-like memory models, and give us an angle from which to attack the problem of structured data. Another natural next step is to integrate our specification into a framework for concurrent memory models, allowing us to instantiate it with realistic models (such as CompCertTSO) that include allocation and free operations and verify optimizations with respect to those models. Ultimately, we aim to construct a unified specification for memory models that can be used to support and simplify any compiler verification effort.

Acknowledgements This work is supported by NSF grants 1065166, 1116682, and 1337174. Any opinions, findings, and conclusions or recommendations expressed in this material are those of the authors and do not necessarily reflect the views of the NSF. 


\section{References}

1. Higham, L., Kawash, J., Verwaal, N.: Defining and comparing memory consistency models. In: In Proc. of the 10th Int'l Conf. on Parallel and Distributed Computing Systems. pp. 349-356 (1997)

2. Kang, J., Hur, C., Mansky, W., Garbuzov, D., Zdancewic, S., Vafeiadis, V.: A formal $\mathrm{C}$ memory model supporting integer-pointer casts. In: ACM SIGPLAN Conference on Programming Language Design and Implementation, PLDI '15 (to appear)

3. Lattner, C., Adve, V.: LLVM: A compilation framework for lifelong program analysis \& transformation. In: Proceedings of the international symposium on Code generation and optimization: feedback-directed and runtime optimization. pp. 75-. CGO '04, IEEE Computer Society, Washington, DC, USA (2004), http: //dl.acm.org/citation.cfm?id=977395.977673

4. Leroy, X.: A formally verified compiler back-end. J. Autom. Reason. 43(4), 363-446 (Dec 2009), http://dx.doi.org/10.1007/s10817-009-9155-4

5. Leroy, X., Blazy, S.: Formal verification of a C-like memory model and its uses for verifying program transformations. J. Autom. Reason. 41, 1-31 (July 2008), http://dl.acm.org/citation. cfm?id=1388522.1388533

6. Owens, S., Sarkar, S., Sewell, P.: A better x86 memory model: x86-TSO. In: Proceedings of the 22nd International Conference on Theorem Proving in Higher Order Logics. pp. 391-407. TPHOLs '09, Springer-Verlag, Berlin, Heidelberg (2009), http://dx.doi.org/10.1007/978-3-642-03359-9_27

7. Saraswat, V.A., Jagadeesan, R., Michael, M., von Praun, C.: A theory of memory models. In: Proceedings of the 12th ACM SIGPLAN Symposium on Principles and Practice of Parallel Programming. pp. 161-172. PPoPP '07, ACM, New York, NY, USA (2007), http://doi.acm.org/10.1145/1229428.1229469

8. Stewart, G., Beringer, L., Cuellar, S., Appel, A.W.: Compositional compcert. In: Proceedings of the $42 \mathrm{Nd}$ Annual ACM SIGPLAN-SIGACT Symposium on Principles of Programming Languages. pp. 275-287. POPL '15, ACM, New York, NY, USA (2015), http://doi.acm.org/10.1145/2676726.2676985

9. Ševčík, J., Vafeiadis, V., Zappa Nardelli, F., Jagannathan, S., Sewell, P.: CompCertTSO: A verified compiler for relaxed-memory concurrency. J. ACM 60(3), 22:1-22:50 (Jun 2013), http://doi.acm.org/10.1145/2487241.2487248

10. Yang, Y., Gopalakrishnan, G., Lindstrom, G., Slind, K.: Nemos: a framework for axiomatic and executable specifications of memory consistency models. In: Parallel and Distributed Processing Symposium, 2004. Proceedings. 18th International. pp. 31- (April 2004)

11. Zhao, J., Nagarakatte, S., Martin, M.M., Zdancewic, S.: Formalizing the LLVM intermediate representation for verified program transformations. SIGPLAN Not. 47(1), 427-440 (Jan 2012), http://doi.acm.org/10.1145/2103621.2103709 